PROCEEDINGS OF THE

AMERICAN MATHEMATICAL SOCIETY

Volume 135, Number 5, May 2007, Pages 1309-1318

S 0002-9939(06)08630-8

Article electronically published on November 14, 2006

\title{
NEW PSEUDORANDOM SEQUENCES CONSTRUCTED BY QUADRATIC RESIDUES AND LEHMER NUMBERS
}

\author{
HUANING LIU
}

(Communicated by Wen-Ching Winnie Li)

Abstract. Let $p$ be an odd prime. Define

$$
e_{n}= \begin{cases}(-1)^{n+\bar{n}}, & \text { if } n \text { is a quadratic residue } \bmod p \\ (-1)^{n+\bar{n}+1}, & \text { if } n \text { is a quadratic nonresidue } \bmod p,\end{cases}
$$

where $\bar{n}$ is the multiplicative inverse of $n$ modulo $p$ such that $1 \leq \bar{n} \leq p-1$. This paper shows that the sequence $\left\{e_{n}\right\}$ is a "good" pseudorandom sequence, by using the properties of exponential sums, character sums, Kloosterman sums and mean value theorems of Dirichlet $L$-functions.

\section{INTRODUCTION}

Let $p$ be an odd prime. For any integer $n$ with $1 \leq n \leq p-1$, we define $\bar{n}$ to be the multiplicative inverse of $n$ modulo $p$ such that $1 \leq \bar{n} \leq p-1$. D. H. Lehmer 7 asked us to study the case that $n$ and $\bar{n}$ are of opposite parity. In [13] and [14] W. Zhang proved that

$$
\sum_{n=1}^{p-1}(-1)^{n+\bar{n}} \ll p^{\frac{1}{2}} \log ^{2} p .
$$

Later he (partly with coauthors) gave a few generalizations on this subject (see [15] 18 for details). Recently S. R. Louboutin, J. Rivat and A. Sárközy [8] showed that the sequence $\left\{(-1)^{n+\bar{n}}\right\}$ forms a "good" pseudorandom sequence.

In a series of papers Mauduit, Rivat and Sárközy (partly with other coauthors) studied finite pseudorandom binary sequences

$$
E_{N}=\left\{e_{1}, \cdots, e_{N}\right\} \in\{-1,+1\}^{N} .
$$

In 9] Mauduit and Sárközy first introduced the following measures of pseudorandomness: the well-distribution measure of $E_{N}$ is defined by

$$
W\left(E_{N}\right)=\max _{a, b, t}\left|\sum_{j=0}^{t-1} e_{a+j b}\right|,
$$

Received by the editors October 28, 2005 and, in revised form, December 23, 2005.

2000 Mathematics Subject Classification. Primary 11A07; 11K45.

Key words and phrases. Pseudorandom, binary sequence, inverse.

This work was supported by the NSF $(10271093,60472068)$ of P. R. China.

(C)2006 American Mathematical Society Reverts to public domain 28 years from publication 
where the maximum is taken over all $a, b, t \in \mathcal{N}$ with $1 \leq a \leq a+(t-1) b \leq N$. The correlation measure of order $k$ of $E_{N}$ is denoted as

$$
C_{k}\left(E_{N}\right)=\max _{M, D}\left|\sum_{n=1}^{M} e_{n+d_{1}} e_{n+d_{2}} \cdots e_{n+d_{k}}\right|,
$$

where the maximum is taken over all $D=\left(d_{1}, \cdots, d_{k}\right)$ and $M$ with $0 \leq d_{1}<\cdots<$ $d_{k} \leq N-M$, and the combined (well-distribution-correlation) PR-measure of order $k$,

$$
Q_{k}\left(E_{N}\right)=\max _{a, b, t, D}\left|\sum_{j=0}^{t} e_{a+j b+d_{1}} e_{a+j b+d_{2}} \cdots e_{a+j b+d_{k}}\right|
$$

is defined for all $a, b, t, D=\left(d_{1}, \cdots, d_{k}\right)$ with $1 \leq a+j b+d_{i} \leq N(i=1,2, \cdots, k)$. In [10] the connection between the measures $W$ and $C_{2}$ was studied.

The sequence is considered as a "good" pseudorandom sequence if both $W\left(E_{N}\right)$ and $C_{k}\left(E_{N}\right)$ (at least for small $k$ ) are "small" in terms of $N$. Later Cassaigne, Mauduit and Sárközy [4 proved that this terminology is justified since for almost all $E_{N} \in\{-1,+1\}^{N}$, both $W\left(E_{N}\right)$ and $C_{k}\left(E_{N}\right)$ are less than $N^{\frac{1}{2}}(\log N)^{c}$. Moreover, it was shown in [9] that the Legendre symbol forms a "good" pseudorandom sequence. In [2] and [3], Cassaigne and coauthors studied the pseudorandomness of the Liouville function, defined as $\lambda(n)=(-1)^{\Omega(n)}(\Omega(n)$ : number of prime factors of $n$ counted with multiplicity) and also of $\gamma(n)=(-1)^{\omega(n)}(\omega(n)$ : number of distinct prime factors of $n$ ). Furthermore, let

$$
K(m, n ; p)=\sum_{a=1}^{p-1} \mathrm{e}\left(\frac{m a+n \bar{a}}{p}\right)
$$

denote the Kloosterman sum, where $\mathrm{e}(y)=\mathrm{e}^{2 \pi i y}$, and $p$ is a prime. Fouvry (with other coauthors) [5] showed that the signs of $K(1, n ; p)$ form a "good" pseudorandom binary sequence.

As was said in [9], the search for new approaches and new constructions should be continued. The purpose of this paper is to give some new examples of pseudorandom sequences. Let

$$
\begin{aligned}
e_{n} & = \begin{cases}(-1)^{n+\bar{n}}, & \text { if } n \text { is a quadratic residue } \bmod p, \\
(-1)^{n+\bar{n}+1}, & \text { if } n \text { is a quadratic nonresidue } \bmod p,\end{cases} \\
& =(-1)^{n+\bar{n}} \chi_{2}(n),
\end{aligned}
$$

where $\chi_{2}$ is the Legendre symbol. We shall prove that the sequence $\left\{e_{n}\right\}$ is a "good" pseudorandom sequence, that is, the following:

Theorem 1.1. Let $p$ be an odd prime, and let $E_{p-1}=\left\{e_{1}, \cdots, e_{p-1}\right\}$ be defined by (1.1). Then we have

$$
\begin{aligned}
& W\left(E_{p-1}\right) \ll p^{\frac{1}{2}} \log ^{2} p ; \\
& C_{2}\left(E_{p-1}\right) \ll p^{\frac{1}{2}} \log ^{3} p ; \\
& Q_{2}\left(E_{p-1}\right) \ll p^{\frac{1}{2}} \log ^{3} p .
\end{aligned}
$$




\section{Some Lemmas}

We need the following lemmas.

Lemma 2.1. Let $p$ be a prime and let $\chi$ be a Dirichlet character modulo $p$. Define the generalized Kloosterman sum $K(m, n, \chi ; p)$ by

$$
K(m, n, \chi ; p)=\sum_{a=1}^{p-1} \chi(a) e\left(\frac{m a+n \bar{a}}{p}\right) .
$$

If $(m, n, p)=1$, then we have

$$
|K(m, n, \chi ; p)| \leq 2 p^{\frac{1}{2}} .
$$

Proof. See reference [11].

Lemma 2.2. Let $p$ be a prime, let $\chi$ be a Dirichlet character modulo $p$, and let $m$ and $n$ be integers with $(n, p)=1$. Then we have

$$
\sum_{a=1}^{p-1}(-1)^{a} \chi(a) e\left(\frac{m a+n \bar{a}}{p}\right) \ll \sqrt{p} \log p .
$$

Proof. From the trigonometric identity

$$
\sum_{u=1}^{p} \mathrm{e}\left(\frac{u n}{p}\right)= \begin{cases}p, & \text { if } p \mid n, \\ 0, & \text { if } p \nmid n,\end{cases}
$$

we have

$$
\begin{aligned}
& \sum_{a=1}^{p-1}(-1)^{a} \chi(a) \mathrm{e}\left(\frac{m a+n \bar{a}}{p}\right) \\
& =\frac{1}{p} \sum_{a=1}^{p-1} \sum_{b=1}^{p-1} \sum_{u=1}^{p} \mathrm{e}\left(\frac{u(a-b)}{p}\right)(-1)^{b} \chi(a) \mathrm{e}\left(\frac{m a+n \bar{a}}{p}\right) \\
& =\frac{1}{p} \sum_{u=1}^{p} \sum_{b=1}^{p-1}(-1)^{b} \mathrm{e}\left(-\frac{u b}{p}\right) \sum_{a=1}^{p-1} \chi(a) \mathrm{e}\left(\frac{(m+u) a+n \bar{a}}{p}\right) .
\end{aligned}
$$

Noting that

$$
\sum_{b=1}^{p-1}(-1)^{b} \mathrm{e}\left(-\frac{u b}{p}\right) \ll \frac{1}{\left|\sin \left(\frac{\pi}{2}-\frac{\pi u}{p}\right)\right|},
$$

then by Lemma 2.1 we get

$$
\sum_{a=1}^{p-1}(-1)^{a} \chi(a) \mathrm{e}\left(\frac{m a+n \bar{a}}{p}\right) \ll \frac{1}{\sqrt{p}} \sum_{u=1}^{p} \frac{1}{\left|\sin \left(\frac{\pi}{2}-\frac{\pi u}{p}\right)\right|} \ll \sqrt{p} \log p .
$$

This proves Lemma 2.2 . 
Lemma 2.3. Let $\Psi$ be a nontrivial additive character, let $\chi$ be a multiplicative character on a finite field $\mathcal{F}_{q}$ of characteristic $p$, let $f, g$ be rational functions in $\mathcal{F}_{q}(x)$ and let

$$
K(\Psi, f ; \chi, g)=\sum_{x \in \mathcal{F}_{q} \backslash s} \chi(g(x)) \Psi(f(x)),
$$

where $s$ denotes the set of poles of $f$ and $g$. For $f=f_{1} / f_{2}$ we define $\operatorname{deg}(f)=$ $\operatorname{deg}\left(f_{1}\right)-\operatorname{deg}\left(f_{2}\right)$. If $K(\Psi, f ; \chi, g)$ is a nondegenerate sum with polynomial $f$ and rational $g$, we have

$$
|K(\Psi, f ; \chi, g)| \leq(\operatorname{deg}(f)+l-1) q^{\frac{1}{2}},
$$

where $l$ is the number of distinct zeros and (non-infinite) poles of $g$ in $\overline{\mathcal{F}}_{p}$.

Proof. See reference [12].

Lemma 2.4. Let $p$ be an odd prime, let $\chi_{2}$ be the Legendre symbol, and let $r$ and $s$ be integers with $(r s, p)=1$. Then for $1 \leq a+t b+d_{2} \leq p-1,0 \leq d_{1}<d_{2}$ and $1 \leq a+d_{1}$, we have

$$
\Lambda=\sum_{j=0}^{t} \chi_{2}\left(a+j b+d_{1}\right) \chi_{2}\left(a+j b+d_{2}\right) \mathrm{e}\left(\frac{r \overline{a+j b+d_{1}}+s \overline{a+j b+d_{2}}}{p}\right) \ll p^{\frac{1}{2}} \log p .
$$

Proof. By (2.1) we get

$$
\begin{aligned}
& \Lambda=\frac{1}{p} \sum_{\substack{j=0 \\
p \nmid a+j b+d_{1} \\
p \nmid a+j b+d_{2}}}^{p-1} \sum_{l=0}^{t} \sum_{u=1}^{p} \mathrm{e}\left(\frac{u(j-l)}{p}\right) \chi_{2}\left(a+j b+d_{1}\right) \chi_{2}\left(a+j b+d_{2}\right) \\
& \times \mathrm{e}\left(\frac{r \overline{a+j b+d_{1}}+s \overline{a+j b+d_{2}}}{p}\right) \\
& =\frac{1}{p} \sum_{u=1}^{p} \sum_{l=0}^{t} \mathrm{e}\left(-\frac{u l}{p}\right) \sum_{\substack{j=0 \\
p \nmid a+j b+d_{1} \\
p \nmid a+j b+d_{2}}}^{p-1} \chi_{2}\left(a+j b+d_{1}\right) \chi_{2}\left(a+j b+d_{2}\right) \\
& \times \mathrm{e}\left(\frac{r \overline{a+j b+d_{1}}+s \overline{a+j b+d_{2}}+u j}{p}\right) .
\end{aligned}
$$

Let $g(j)=\left(a+j b+d_{1}\right)\left(a+j b+d_{2}\right)$ and

$$
f(j)=\frac{r\left(a+j b+d_{2}\right)+s\left(a+j b+d_{1}\right)+u j\left(a+j b+d_{1}\right)\left(a+j b+d_{2}\right)}{\left(a+j b+d_{1}\right)\left(a+j b+d_{2}\right)} .
$$

Then the sum

$$
\begin{aligned}
& \sum_{\substack{j=0 \\
p \nmid a+j b+d_{1} \\
p \nmid a+j b+d_{2}}}^{p-1} \chi_{2}\left(a+j b+d_{1}\right) \chi_{2}\left(a+j b+d_{2}\right) \mathrm{e}\left(\frac{r \overline{a+j b+d_{1}}+s \overline{a+j b+d_{2}}+u j}{p}\right) \\
& =\sum_{\substack{j=0 \\
p \nmid a+j b+d_{1} \\
p \nmid a+j b+d_{2}}}^{p-1} \chi_{2}(g(x)) \mathrm{e}\left(\frac{f(x)}{p}\right)
\end{aligned}
$$


is nondegenerate since $(r s, p)=1$ and $d_{1} \neq d_{2}$. Noting that

$$
\sum_{l=0}^{t} \mathrm{e}\left(-\frac{u l}{p}\right) \ll \frac{1}{\left|\sin \left(\frac{\pi u}{p}\right)\right|}, \quad \text { for } p \nmid u
$$

then from Lemma 2.3 we have

$$
\Lambda \ll \frac{t}{\sqrt{p}}+\frac{1}{\sqrt{p}} \sum_{u=1}^{p-1} \frac{1}{\left|\sin \left(\frac{\pi u}{p}\right)\right|} \ll p^{\frac{1}{2}} \log p
$$

This completes the proof of Lemma 2.4.

Lemma 2.5. Let $p$ be an odd prime and let $k_{1}$ and $k_{2}$ be nonnegative integers. Then for $1 \leq a+t b+d_{2} \leq p-1,0 \leq d_{1}<d_{2}$ and $1 \leq a+d_{1}$, we have

$$
\begin{aligned}
\Upsilon= & \sum_{j=0}^{t} \chi_{2}\left(a+j b+d_{1}\right) \chi_{2}\left(a+j b+d_{2}\right) \sum_{\substack{\chi^{\prime}(-1)=-1 \\
\chi^{\prime} \bmod p}} \sum_{\substack{\chi^{\prime \prime}(-1)=-1 \\
\chi^{\prime \prime} \bmod p}} \chi^{\prime}\left(2^{k_{1}}\right) \chi^{\prime \prime}\left(2^{k_{2}}\right) \\
& \times \chi^{\prime}\left(a+j b+d_{1}\right) \chi^{\prime \prime}\left(a+j b+d_{2}\right) \tau\left(\chi^{\prime}\right) \tau\left(\chi^{\prime \prime}\right) L\left(1, \bar{\chi}^{\prime}\right) L\left(1, \bar{\chi}^{\prime \prime}\right) \\
\ll & p^{\frac{5}{2}} \log ^{3} p,
\end{aligned}
$$

where $\chi_{2}$ is the Legendre symbol, $\tau(\chi)=\sum_{a=1}^{p-1} \chi(a) e\left(\frac{a}{p}\right)$ is the Gauss sum, and $L(1, \chi)$ denotes the Dirichlet $L$-function.

Proof. For any nonprincipal character $\chi$ modulo $p$, and parameter $N \geq p$, by Abel's identity we get

$$
\begin{aligned}
L(1, \chi) & =\sum_{n=1}^{+\infty} \frac{\chi(n)}{n}=\sum_{1 \leq n \leq N} \frac{\chi(n)}{n}+\int_{N}^{+\infty} \frac{\sum_{N<n \leq y} \chi(n)}{y^{2}} \mathrm{~d} y \\
& =\sum_{1 \leq n \leq N} \frac{\chi(n)}{n}+O\left(\frac{\sqrt{p} \log p}{N}\right)
\end{aligned}
$$


Then we have

$$
\begin{aligned}
& \Upsilon=\sum_{j=0}^{t} \chi_{2}\left(a+j b+d_{1}\right) \chi_{2}\left(a+j b+d_{2}\right) \sum_{\substack{\chi^{\prime}(-1)=-1 \\
\chi^{\prime} \bmod p \quad \chi^{\prime \prime}(-1)=-1 \\
\chi^{\prime \prime} \bmod p}} \chi^{\prime}\left(2^{k_{1}}\right) \chi^{\prime \prime}\left(2^{k_{2}}\right) \\
& \times \chi^{\prime}\left(a+j b+d_{1}\right) \chi^{\prime \prime}\left(a+j b+d_{2}\right) \sum_{r=1}^{p-1} \chi^{\prime}(r) \mathrm{e}\left(\frac{r}{p}\right) \sum_{s=1}^{p-1} \chi^{\prime \prime}(s) \mathrm{e}\left(\frac{s}{p}\right) \\
& \times \sum_{1 \leq n \leq N} \frac{\bar{\chi}^{\prime}(n)}{n} \sum_{1 \leq m \leq N} \frac{\bar{\chi}^{\prime \prime}(m)}{m}+O\left(\frac{t p^{\frac{7}{2}} \log p \log N}{N}\right) \\
& =\sum_{j=0}^{t} \chi_{2}\left(a+j b+d_{1}\right) \chi_{2}\left(a+j b+d_{2}\right) \sum_{1 \leq n \leq N} \frac{1}{n} \sum_{1 \leq m \leq N} \frac{1}{m} \sum_{r=1}^{p-1} \mathrm{e}\left(\frac{r}{p}\right) \sum_{s=1}^{p-1} \mathrm{e}\left(\frac{s}{p}\right) \\
& \times \sum_{\substack{\chi^{\prime}(-1)=-1 \\
\chi^{\prime} \bmod p}} \chi^{\prime}\left(2^{k_{1}}\right) \chi^{\prime}\left(a+j b+d_{1}\right) \chi^{\prime}(r) \bar{\chi}^{\prime}(n) \\
& \times \sum_{\substack{\chi^{\prime \prime}(-1)=-1 \\
\chi^{\prime \prime} \bmod p}} \chi^{\prime \prime}\left(2^{k_{2}}\right) \chi^{\prime \prime}\left(a+j b+d_{2}\right) \chi^{\prime \prime}(s) \bar{\chi}^{\prime \prime}(m)+O\left(\frac{t p^{\frac{7}{2}} \log p \log N}{N}\right) \\
& \text { (2.4) }=\Omega+O\left(\frac{t p^{\frac{7}{2}} \log p \log N}{N}\right) \text {. }
\end{aligned}
$$

For $(a b, p)=1$, from the orthogonality relation for character sums,

$$
\sum_{\chi \bmod p} \chi(a) \bar{\chi}(b)= \begin{cases}p-1, & \text { if } a \equiv b \bmod p \\ 0, & \text { otherwise }\end{cases}
$$

we get

$$
\sum_{\substack{\chi(-1)=-1 \\ \chi \bmod p}} \chi(a) \bar{\chi}(b)= \begin{cases}\frac{1}{2}(p-1), & \text { if } a \equiv b \bmod p \\ -\frac{1}{2}(p-1), & \text { if } a \equiv-b \bmod p \\ 0, & \text { otherwise }\end{cases}
$$

Therefore

$$
\begin{gathered}
\Omega=\frac{(p-1)^{2}}{4} \sum_{j=0}^{t} \chi_{2}\left(a+j b+d_{1}\right) \chi_{2}\left(a+j b+d_{2}\right) \sum_{\substack{1 \leq n \leq N \\
(n, p)=1}} \frac{1}{n} \sum_{\substack{1 \leq m \leq N \\
(m, p)=1}} \frac{1}{m} \\
\times \sum_{\substack{r=1 \\
2^{k_{1}}\left(a+j b+d_{1}\right) r \equiv n \bmod p}}^{p-1} \mathrm{e}\left(\frac{r}{p}\right) \sum_{\substack{s=1 \\
2^{k_{2}}\left(a+j b+d_{2}\right) s \equiv m \bmod p}}^{p-1} \mathrm{e}\left(\frac{s}{p}\right)
\end{gathered}
$$




$$
\begin{aligned}
& -\frac{(p-1)^{2}}{4} \sum_{j=0}^{t} \chi_{2}\left(a+j b+d_{1}\right) \chi_{2}\left(a+j b+d_{2}\right) \sum_{\substack{1 \leq n \leq N \\
(n, p)=1}} \frac{1}{n} \sum_{\substack{1 \leq m \leq N \\
(m, p)=1}} \frac{1}{m} \\
& \times \quad \sum_{r=1}^{p-1} \mathrm{e}\left(\frac{r}{p}\right) \quad \sum_{s=1}^{p-1} \mathrm{e}\left(\frac{s}{p}\right) \\
& 2^{k_{1}}\left(a+j b+d_{1}\right) r \equiv n \bmod p \quad 2^{k_{2}}\left(a+j b+d_{2}\right) s \equiv-m \bmod p \\
& -\frac{(p-1)^{2}}{4} \sum_{j=0}^{t} \chi_{2}\left(a+j b+d_{1}\right) \chi_{2}\left(a+j b+d_{2}\right) \sum_{\substack{1 \leq n \leq N \\
(n, p)=1}} \frac{1}{n} \sum_{\substack{1 \leq m \leq N \\
(m, p)=1}} \frac{1}{m}
\end{aligned}
$$

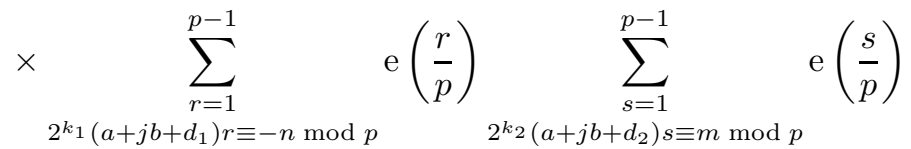

$$
\begin{aligned}
& +\frac{(p-1)^{2}}{4} \sum_{j=0}^{t} \chi_{2}\left(a+j b+d_{1}\right) \chi_{2}\left(a+j b+d_{2}\right) \sum_{\substack{1 \leq n \leq N \\
(n, p)=1}} \frac{1}{n} \sum_{\substack{1 \leq m \leq N \\
(m, p)=1}} \frac{1}{m} \\
& \times \quad \sum_{r=1}^{p-1} \quad \mathrm{e}\left(\frac{r}{p}\right) \quad \sum_{s=1}^{p-1} \mathrm{e}\left(\frac{s}{p}\right) \\
& 2^{k_{1}}\left(a+j b+d_{1}\right) r \equiv-n \bmod p \quad 2^{k_{2}}\left(a+j b+d_{2}\right) s \equiv-m \bmod p \\
& =\Omega_{1}+\Omega_{2}+\Omega_{3}+\Omega_{4} \text {. }
\end{aligned}
$$

By Lemma 2.4 we have

$$
\begin{aligned}
\Omega_{1}= & \frac{(p-1)^{2}}{4} \sum_{\substack{1 \leq n \leq N \\
(n, p)=1}} \frac{1}{n} \sum_{\substack{1 \leq m \leq N \\
(m, p)=1}} \frac{1}{m} \sum_{j=0}^{t} \chi_{2}\left(a+j b+d_{1}\right) \chi_{2}\left(a+j b+d_{2}\right) \\
& \times \mathrm{e}\left(\frac{n \overline{2^{k_{1}}} \overline{a+j b+d_{1}}+m \overline{2^{k_{2}}} \overline{a+j b+d_{2}}}{p}\right) \\
\ll & \ll p^{\frac{5}{2}} \log p \log ^{2} N .
\end{aligned}
$$

Similarly we get

$$
\Omega_{2}, \Omega_{3}, \Omega_{4} \ll p^{\frac{5}{2}} \log p \log ^{2} N
$$

Now taking $N=p^{2}$ in (2.4), (2.6), (2.7) and (2.8), we immediately have

$$
\Upsilon \ll p^{\frac{5}{2}} \log ^{3} p
$$

This proves Lemma 2.5. 


\section{Proof of the theorem}

For $a, b, t$ with $1 \leq a \leq a+(t-1) b \leq p-1$, by (1.1) and (2.1) we have

$$
\begin{aligned}
& \sum_{j=0}^{t-1} e_{a+j b}=\sum_{j=0}^{t-1}(-1)^{a+j b+\overline{a+j b}} \chi_{2}(a+j b) \\
& =\frac{1}{p^{3}} \sum_{\substack{j=0 \\
p \nmid a+j b}}^{p-1} \sum_{l=0}^{t-1} \sum_{u=1}^{p} \mathrm{e}\left(\frac{u(j-l)}{p}\right) \sum_{c=1}^{p-1} \sum_{r=1}^{p} \mathrm{e}\left(\frac{r(a+j b-c)}{p}\right) \\
& \times \sum_{d=1}^{p-1} \sum_{s=1}^{p} \mathrm{e}\left(\frac{s(\overline{a+j b}-d)}{p}\right)(-1)^{c+d} \chi_{2}(c) \\
& =\frac{1}{p^{3}} \sum_{r=1}^{p} \sum_{s=1}^{p-1} \sum_{u=1}^{p} \sum_{l=0}^{t-1} \mathrm{e}\left(-\frac{u l}{p}\right) \sum_{c=1}^{p-1}(-1)^{c} \chi_{2}(c) \mathrm{e}\left(-\frac{r c}{p}\right) \sum_{d=1}^{p-1}(-1)^{d} \mathrm{e}\left(-\frac{s d}{p}\right) \\
& \times \sum_{\substack{j=0 \\
p \nmid a+j b}}^{p-1} \mathrm{e}\left(\frac{r(a+j b)+s \overline{a+j b}+u j}{p}\right) \\
& =\frac{1}{p^{3}} \sum_{r=1}^{p} \sum_{s=1}^{p-1} \sum_{u=1}^{p} \sum_{l=0}^{t-1} \mathrm{e}\left(-\frac{u l}{p}\right) \sum_{c=1}^{p-1}(-1)^{c} \chi_{2}(c) \mathrm{e}\left(-\frac{r c}{p}\right) \sum_{d=1}^{p-1}(-1)^{d} \mathrm{e}\left(-\frac{s d}{p}\right) \\
& \times \mathrm{e}\left(-\frac{u a \bar{b}}{p}\right) \sum_{t=1}^{p-1} \mathrm{e}\left(\frac{r t+s \bar{t}+u \bar{b} t}{p}\right) \\
& =\frac{1}{p^{2}} \sum_{s=1}^{p-1}\left(\sum_{d=1}^{p-1}(-1)^{d} \mathrm{e}\left(-\frac{s d}{p}\right)\right) \sum_{u=1}^{p}\left(\sum_{l=0}^{t-1} \mathrm{e}\left(-\frac{u l}{p}\right)\right) \mathrm{e}\left(-\frac{u a \bar{b}}{p}\right) \\
& \times\left(\sum_{t=1}^{p-1}(-1)^{t} \chi_{2}(t) \mathrm{e}\left(\frac{u \bar{b} t+s \bar{t}}{p}\right)\right) .
\end{aligned}
$$

Then by (2.2), (2.3) and Lemma 2.2 we get

$$
\begin{aligned}
\sum_{j=0}^{t-1} e_{a+j b} & \ll \frac{t p^{\frac{1}{2}}}{p^{2}} \sum_{s=1}^{p-1} \frac{1}{\left|\sin \left(\frac{\pi}{2}-\frac{\pi s}{p}\right)\right|}+\frac{p^{\frac{1}{2}}}{p^{2}} \sum_{s=1}^{p-1} \frac{1}{\left|\sin \left(\frac{\pi}{2}-\frac{\pi s}{p}\right)\right|} \sum_{u=1}^{p-1} \frac{1}{\left|\sin \left(\frac{\pi u}{p}\right)\right|} \\
& \ll p^{\frac{1}{2}} \log ^{2} p .
\end{aligned}
$$

Therefore

$$
W\left(E_{p-1}\right)=\max _{a, b, t}\left|\sum_{j=0}^{t-1} e_{a+j b}\right| \ll p^{\frac{1}{2}} \log ^{2} p .
$$


For $1 \leq a+t b+d_{2} \leq p-1$ and $0 \leq d_{1}<d_{2}$, by (1.1) and (2.5) we have

$$
\begin{aligned}
& \sum_{j=0}^{t} e_{a+j b+d_{1}} e_{a+j b+d_{2}} \\
& =\sum_{j=0}^{t}(-1)^{a+j b+d_{1}+\overline{a+j b+d_{1}}} \chi_{2}\left(a+j b+d_{1}\right)(-1)^{a+j b+d_{2}+\overline{a+j b+d_{2}}} \chi_{2}\left(a+j b+d_{2}\right) \\
& =(-1)^{d_{1}+d_{2}} \sum_{j=0}^{t}(-1)^{\overline{a+j b+d_{1}}+\overline{a+j b+d_{2}}} \chi_{2}\left(a+j b+d_{1}\right) \chi_{2}\left(a+j b+d_{2}\right) \\
& =\frac{(-1)^{d_{1}+d_{2}}}{(p-1)^{2}} \sum_{j=0}^{t} \sum_{r=1}^{p-1} \sum_{s=1}^{p-1} \sum_{\chi^{\prime} \bmod p} \chi^{\prime}\left(r\left(a+j b+d_{1}\right)\right) \sum_{\chi^{\prime \prime} \bmod p} \chi^{\prime \prime}\left(s\left(a+j b+d_{2}\right)\right) \\
& \times(-1)^{r+s} \chi_{2}\left(a+j b+d_{1}\right) \chi_{2}\left(a+j b+d_{2}\right) \\
& =\frac{(-1)^{d_{1}+d_{2}}}{(p-1)^{2}} \sum_{\chi^{\prime} \bmod p} \sum_{\chi^{\prime \prime} \bmod p} \sum_{j=0}^{t}\left(\chi^{\prime}\left(a+j b+d_{1}\right) \chi_{2}\left(a+j b+d_{1}\right)\right. \\
& \left.\times \chi^{\prime \prime}\left(a+j b+d_{2}\right) \chi_{2}\left(a+j b+d_{2}\right)\right) \\
& \times \sum_{r=1}^{p-1}(-1)^{r} \chi^{\prime}(r) \sum_{s=1}^{p-1}(-1)^{s} \chi^{\prime \prime}(s) .
\end{aligned}
$$

Noting that

$$
\sum_{r=1}^{p-1}(-1)^{r} \chi(r)=0, \quad \text { if } \chi(-1)=1,
$$

while if $\chi(-1)=-1$, from [6] and Theorem 12.11, 12.20 of [1] we have

$$
\begin{aligned}
\sum_{r=1}^{p-1}(-1)^{r} \chi(r) & =2 \chi(2) \sum_{r=1}^{(p-1) / 2} \chi(r)=\frac{2(1-2 \chi(2))}{p} \sum_{r=1}^{p-1} r \chi(r) \\
& =\frac{2(1-2 \chi(2)) i}{\pi} \tau(\chi) L(1, \bar{\chi}) .
\end{aligned}
$$

So from Lemma 2.5 we get

$$
\begin{aligned}
& \sum_{j=0}^{t} e_{a+j b+d_{1}} e_{a+j b+d_{2}}=\frac{4(-1)^{d_{1}+d_{2}+1}}{\pi^{2}(p-1)^{2}} \sum_{j=0}^{t} \chi_{2}\left(a+j b+d_{1}\right) \chi_{2}\left(a+j b+d_{2}\right) \\
& \quad \times \sum_{\substack{\chi^{\prime}(-1)=-1 \\
\chi^{\prime} \bmod p}} \sum_{\chi^{\prime \prime}(-1)=-1}\left(1-2 \chi^{\prime}(2)\right)\left(1-2 \chi^{\prime \prime}(2)\right) \chi^{\prime}\left(a+j b+d_{1}\right) \chi^{\prime \prime}\left(a+j b+d_{2}\right) \\
& \times \quad \tau\left(\chi^{\prime}\right) \tau\left(\chi^{\prime \prime}\right) L\left(1, \bar{\chi}^{\prime}\right) L\left(1, \bar{\chi}^{\prime \prime}\right) \\
& \ll p^{\frac{1}{2}} \log ^{3} p .
\end{aligned}
$$

Therefore

$$
Q_{2}\left(E_{p-1}\right)=\max _{a, b, t, D}\left|\sum_{j=0}^{t} e_{a+j b+d_{1}} e_{a+j b+d_{2}}\right| \ll p^{\frac{1}{2}} \log ^{3} p .
$$


Now taking $a=0, b=1, j=n-1$ and $t=M-1$ in (3.1), we immediately get

$$
C_{2}\left(E_{p-1}\right)=\max _{M, D}\left|\sum_{n=1}^{M} e_{n+d_{1}} e_{n+d_{2}}\right| \ll p^{\frac{1}{2}} \log ^{3} p .
$$

This completes the proof of Theorem 1.1.

\section{ACKNOWLEDGMENTS}

The author expresses his gratitude to the referee for his detailed comments.

\section{REFERENCES}

[1] T. M. Apostol, Introduction to analytic number theory, Springer-Verlag, New York, 1976. MR0434929 (55:7892)

[2] J. Cassaigne, S. Ferenczi, C. Mauduit, J. Rivat and A. Sárközy, On finite pseudorandom binary sequencs III: the Liouville function, I, Acta Arithmetica, 87 (1999), pp. 367-390. MR.1671629 (2000c:11126)

[3] J. Cassaigne, S. Ferenczi, C. Mauduit, J. Rivat and A. Sárközy, On finite pseudorandom binary sequencs IV: the Liouville function, II, Acta Arithmetica, 95 (2000), pp. 343-359. MR:1785199 (2002c:11087)

[4] J. Cassaigne, C. Mauduit and A. Sárközy, On finite pseudorandom binary sequencs VII: the measures of pseudorandomness, Acta Arithmetica, 103 (2002), pp. 97-108. MR 1904866 (2004c:11139)

[5] E. Fouvry, P. Michel, J. Rivat and A. Sárközy, On the pseudorandomness of the signs of Kloosterman sums, Journal of the Australian Mathematical Society, 77 (2004), pp. 425-436. MR2099811 (2005h:11165)

[6] T. Funakura, On Kronecker's limit formula for Dirichlet series with periodic coefficients, Acta Arithmetica, 55 (1990), pp. 59-73. MR1056115 (91j:11064)

[7] R. K. Guy, Unsolved problems in number theory, Springer-Verlag, New York, 1994. MR.1299330 (96e:11002)

[8] S. R. Louboutin, J. Rivat and A. Sárközy, On a problem of D. H. Lehmer, Proceedings of the American Mathematical Society, to appear.

[9] C. Mauduit and A. Sárközy, On finite pseudorandom binary sequences I: measure of pseudorandomness, the Legendre symbol, Acta Arithmetica, 82 (1997), pp. 365-377. MR1483689 (99g:11095)

[10] C. Mauduit and A. Sárközy, On the measures of pseudorandomness of binary sequences, Discrete Mathematics, 271 (2003), pp. 195-207. MR.1999543 (2004e:11081)

[11] A. Weil, Sur les courbes algébriques et les variétés qui s'en déduisent, Act. Sci. Ind., Vol. 1041, Hermann, Paris, 1948. MR0027151(10:262c)

[12] A. Weil, On some exponential sums, Proc. Nat. Acad. Sci., 34 (1948), pp. 204-207. MR0027006 (10:234e)

[13] W. Zhang, A problem of D. H. Lehmer and its generalization (I), Compositio Mathematica, 86 (1993), pp. 307-316. MR1219630 (94f:11104)

[14] W. Zhang, A problem of D. H. Lehmer and its generalization (II), Compositio Mathematica, 91 (1994), pp. 47-56. MR.1273925 (95f:11079)

[15] W. Zhang, On the difference between a D. H. Lehmer number and its inverse modulo $q$, Acta Arithmetica, 68 (1994), pp. 255-263. MR.1308126 (96a:11100)

[16] W. Zhang, On a problem of D. H. Lehmer and Kloosterman sums, Monatshefte für Mathematik, 139 (2003), pp. 247-257. MR.1994384(2004e:11088)

[17] W. Zhang, On a problem of D. H. Lehmer and general Kloosterman sums, Acta Mathematica Sinica English Series, 20 (2004), pp. 515-524. MR2084715 (2005h:11226)

[18] W. Zhang, Z. Xu and Y. Yi, A problem of D. H. Lehmer and its mean square value formula, Journal of Number Theory, 103 (2003), pp. 197-213. MR2020268 (2004m:11128)

Department of Mathematics, Northwest University, Xi'an, Shannxi, People's RepubLIC OF CHINA

E-mail address: hnliu@nwu.edu.cn 